\title{
LAND SURVEY FROM UNMANED AERIAL VEICHLE
}

\author{
Vid Peterman, Marko Mesarič \\ University of Ljubljana, Faculty of civil and geodetic engineering, Ljubljana, Slovenia \\ vid.peterman@gmail.com
}

KEY WORDS: Low altitude, Three-Dimensional, Non-metric camera, UAVs, Point Cloud

\begin{abstract}
:
In this paper we present, how we use a quadrocopter unmanned aerial vehicle with a camera attached to it, to do low altitude photogrammetric land survey. We use the quadrocopter to take highly overlapping photos of the area of interest. A "structure from motion" algorithm is implemented to get parameters of camera orientations and to generate a sparse point cloud representation of objects in photos. Than a patch based multi view stereo algorithm is applied to generate a dense point cloud. Ground control points are used to georeference the data. Further processing is applied to generate digital orthophoto maps, digital surface models, digital terrain models and assess volumes of various types of material. Practical examples of land survey from a UAV are presented in the paper. We explain how we used our system to monitor the reconstruction of commercial building, then how our UAV was used to assess the volume of coal supply for Ljubljana heating plant. Further example shows the usefulness of low altitude photogrammetry for documentation of archaeological excavations. In the final example we present how we used our UAV to prepare an underlay map for natural gas pipeline's route planning. In the final analysis we conclude that low altitude photogrammetry can help bridge the gap between laser scanning and classic tachymetric survey, since it offers advantages of both techniques.
\end{abstract}

\section{INTRODUCTION}

In March 2011 I received my diploma from the University of Ljubljana, Faculty of Civil and Geodetic Engineering, where I was studying geodetic engineering. During my studies I learnt of a great potential for photogrammetric measurements. After my graduation I wondered could we use image matching based photogrammetric measurements for the purpose of every-day geodetic survey, which could be competed against the use of terrestrial laser scanners and total stations.

With my classmate from the faculty we started a new company. We bought a quad-copter unmanned aerial vehicle (abbreviation UAV) and mounted a digital camera on it. With it we propose a new technique in land survey.

The idea itself for Land survey from the UAV came from 2010 ISPRS symposium in Newcastle where a great number of papers concerning UAV based measurements were presented.

In this paper the emphasis will be on practical examples of UAV based photogrammetric measurements, and, focus on the advantages of photogrammetric measurements over classical geodetic survey.

First, we will use an example to show our approach to low altitude photogrammetry and explain in detail how our system works and how we used it to monitor the construction site of a commercial building reconstruction.

Later in the paper, further examples will be presented. Let us show you how we used low altitude photogrammetric measurements to assess the volume of coal supplies for Ljubljana heating plant, then, how we made ortho-photo map with ground pixel size of $1 \mathrm{~cm}$, point cloud representation and a digital surface model of medieval archaeological excavations.

\section{HARDWARE USED FOR DATA ACQUISITION}

To obtain aerial images we use a Microdrones MD4-1000 quadrocopter with an Olympus EP-1 camera mounted on in. The camera has an $18 \mathrm{~mm} 12$ Megapixel sensor and a fixed length camera lens with a focal distance of $17 \mathrm{~mm}$.

For georeferencing we use ground control points. They are signalized by black circular targets on a white background. To determine positions of ground control points we use a GNSS RTK system or a total station, depending on a situation.

\section{EXAMPLE OF CONSTRUCTION SITE MONITORING}

\subsection{Description of the construction site}

The construction site is located on "Barje" - a wetland region in the southern part of Ljubljana. A decade ago a number of shopping centres were built in the area. Recently, one of the supermarkets began to sink into the ground and a complete reconstruction was needed. As surveyors, our job was to provide documentation, periodical control measurements of reconstruction progress, and to assess volumes of excavated material.

At the time of the writing of the paper, five successive measurements were carried out. As the reconstruction progresses additional measurements will be performed. 


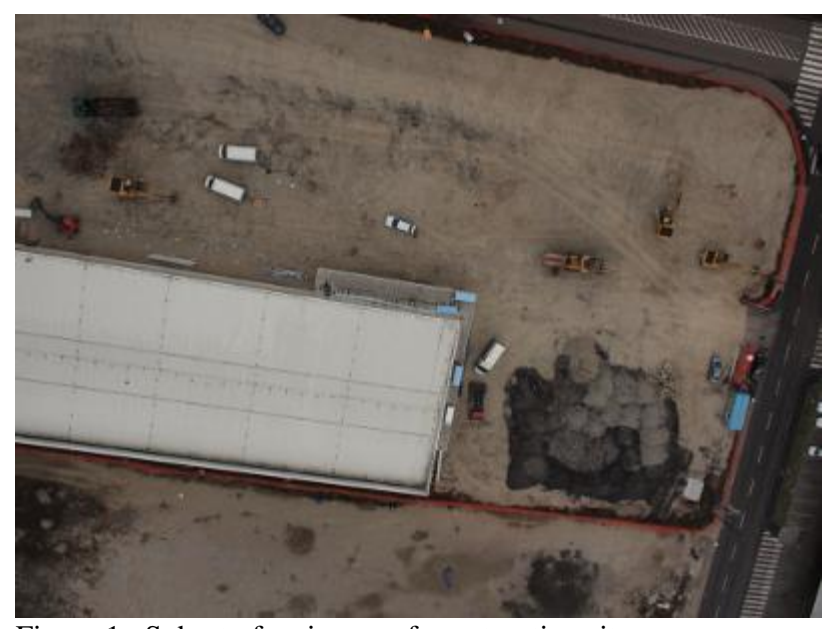

Figure 1. Subset of an image of construction site

\subsection{Data acquisition}

Two persons were required for data acquisition for each of the periodical measurements, a GNSS operator and a quadrocopter pilot. GNSS operator selected the positions for nine ground control points. He signalized them with black circular targets on white background, and determined their positions in Slovene coordinate system using a GNSS rover RTK system.

Meanwhile, the quadrocopter pilot prepared the UAV for takeoff. When everything was prepared, the work on the construction site was halted. The pilot manually lifted the UAV into the air. Once on the safe altitude, he switched the UAV from manual to autonomous mode, and the UAV began its preprogrammed 15 minute path over the construction site.

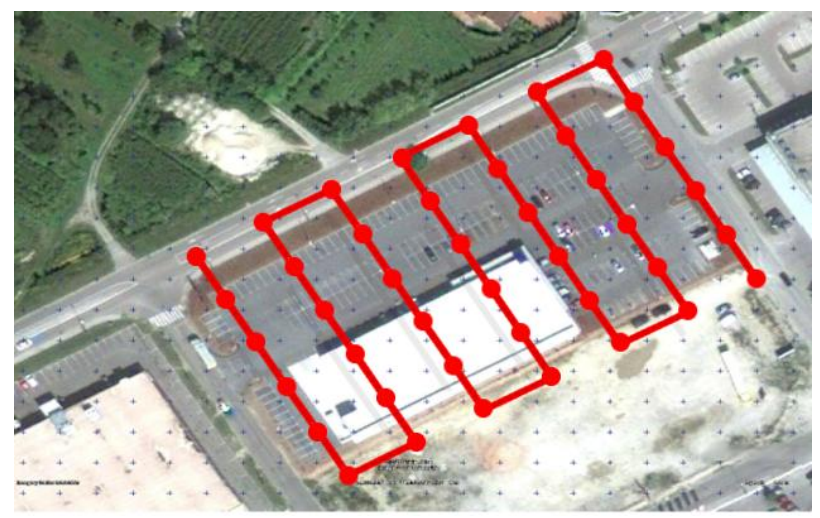

Figure 2. UAV's flight path

Altogether, the UAV took 42 images from the altitude of 80 meters. The images had an overlap of $66 \%$ both in the vertical and horizontal direction.

\subsection{Image and data processing}

To process blocks of images we implemented a modified version of multi view stereo (Niethammer et al., 2010). The method originates from the field of computer vision, and it offers efficient alternative to commercial photogrammetry software.

Our modified method relies on a structure from motion algorithm (Snavely et al., 2008) for a computation of extrinsic and intrinsic parameters for each of supplied photos. Computed model coordinates of projective centres of camera positions, and 3D terrestrial coordinates of quadrocopter positions at the time of image registration, determined by the on-board GPS receiver are then used to calculate the approximate transformation from model coordinates generated by surface to motion algorithm to Slovene coordinate system.

An inverse transformation of ground control points' positions to model coordinates is then computed using approximate transformation parameters. Ground control points' model coordinates are then transformed into image coordinate system for each of camera positions. Following these transformations, we obtain approximate positions of ground control points in each image. Synthetic target adaptive least squares image matching is used to calculate precise model coordinates of ground control points. These in turn combined with terrestrial coordinates of ground control points give precise parameters of transformation between model and Slovene terrestrial coordinate system.

We implemented the process described above into program code, with which we elegantly georeference photogrammetric measurements without the need for manual measurements of image coordinates of ground control points.

A patch-based multi view stereo algorithm (Furukawa et al., 2007 ) is then used. It computes a dense set of small rectangular patches that cover space visible in photos using supplied parameters from structure from motion algorithm. Stereo reconstruction is implemented as matching, expansion and filtering procedure. The result is a dense point cloud representation of recorded scenery.

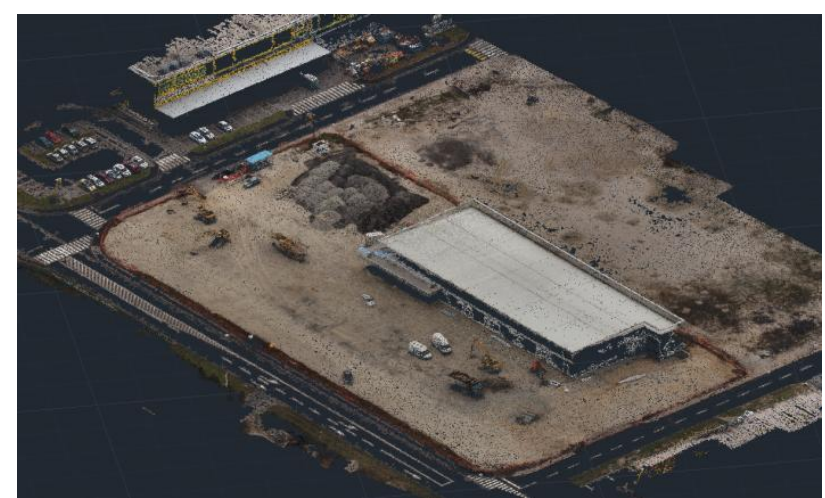

Figure 3. Point cloud representation of construction site area

We use point cloud data to generate a digital terrain model and ortho-photo maps. With comparison of DTM from successive measurements, volumes of excavated and transported material can be assessed. 


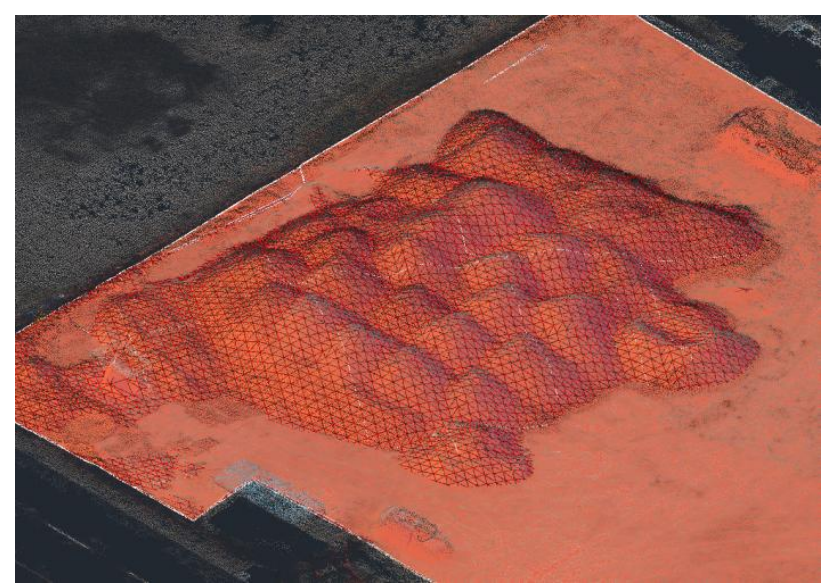

Figure 4. Comparison of DTMs for volume of asphalt pile assessment

\subsection{Accuracy assessment}

For the purpose of accuracy assessment independent control measurements with a total station were conducted. Photogrammetric measurements were compared against control measurements.

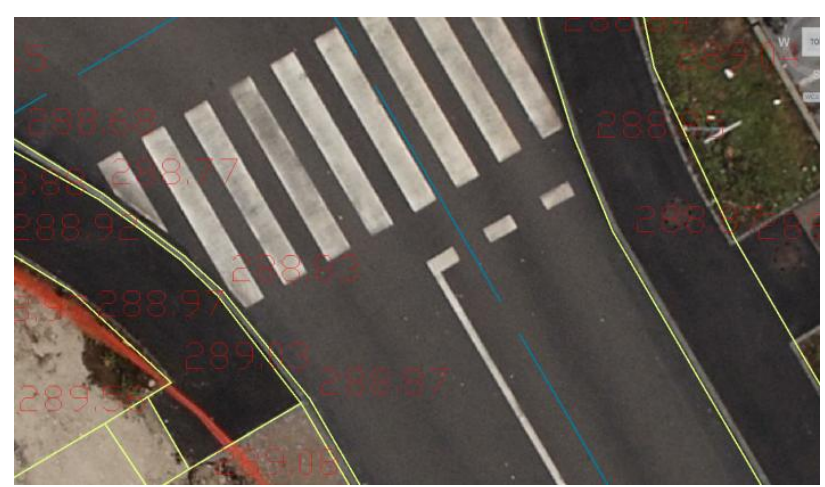

Figure 5. A subset of comparison of photogrammetric (orthophoto) and control measurements

Based on the comparison the accuracy of photogrammetric measurements was assessed to be $2 \mathrm{~cm}$ in horizontal direction and $3 \mathrm{~cm}$ in vertical direction.

\subsection{Summary}

Each of the periodical photogrammetric measurements took approximately 60 minutes (including quadrocopter packing, unpacking and, ground control points signalization and measuring). That is considerably less than classical measurements with a total station or a terrestrial laser scanning would require. Moreover, since terrestrial laser scanners are quite expensive, photogrammetric measurements are a lot cheaper than laser scanning.

Resulting point cloud consists of a couple of millions of points, far more than we can measure with a total station and, therefore, enables precise digital terrain model estimation.

\section{FURTHER EXAMPLES OF LAND SURVEY FROM A UAV}

\subsection{Estimation of volumes}

Low altitude photogrammetry proves very useful for measuring shapes of various piles of material. May it either be coal, sand, earth, rocks or anything of the sort, photos of it have a nice random texture and image matching algorithms work very well with it. If shape is measured at different times, volume changes can be calculated.

Furthermore, we will describe how we used our UAV system at Ljubljana Heating plant. The plant requires a yearly estimation of volume of coal supply. Coal is stored in two piles, measuring approximately $80 \mathrm{~m}$ by $150 \mathrm{~m}$ each.

Until now tachymetric geodetic measurements by a total station were used for generation of digital terrain model of coal supply. To define the shape of piles a $2 \mathrm{~m}$ by $2 \mathrm{~m}$ gird of points were signalised and measured. Such measurements took 2 days to complete. A total number of approximately 6000 points were determined in the process.

On the other hand, this year's photogrammetric measurements only took about three hours to complete. In this time 9 ground control points had been signalized and had its positions determined, plus 130 images have been recorded by our UAV system, and even some additional tachymetric control measurements by total station had been made to help assess the accuracy of generated DTM. The images were recorded from the height of $80 \mathrm{~m}$ and had $66 \%$ overlap in vertical and horizontal direction.

Resulting point cloud consisted of 8 million points. From this point cloud a digital terrain model was computed. DTM grid size was selected to be $0.5 \mathrm{~m}$. With a comparison of photogrammetric and control measurements an accuracy of DTM was assessed to be $4 \mathrm{~cm}$.

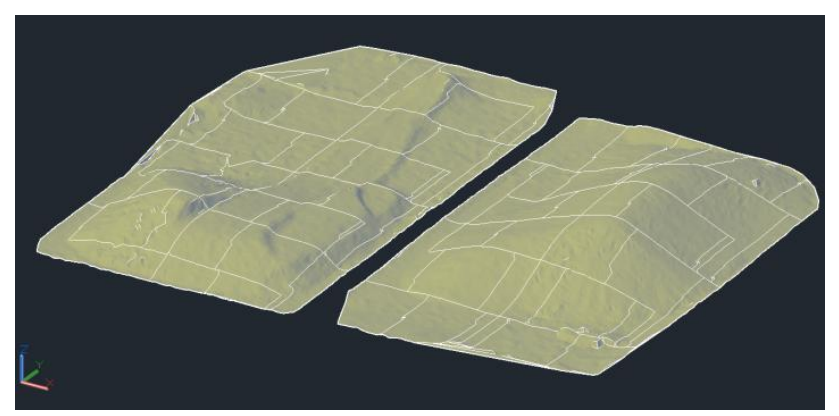

Figure 6. Digital terrain models of heating plant's coal supply

Photogrammetric measurements took considerably less time than classical measurements took in the past. However, the measured point density had been far greater. That is why we were able to generate a DTM with a smaller cell size and elegantly measure the volume of coal supply.

\subsection{Documentation of archaeological excavations}

Low altitude photogrammetry is also very suitable for digital surface model generation. As such it is quite useful for documentation of archaeological excavations.

We had a privilege to work with a group of archaeologists, who had discovered ruins of a medieval castle on a hill above the 
city of Maribor. We used our UAV system to document their work.

Once the excavations had been finished, we took altogether 230 photos of the ruins from the altitude of $30 \mathrm{~m}$ above ground. We chose to fly so low, so that average pixel size in recorded photos would be less than $1 \mathrm{~cm}$ big (approx. $0.75 \mathrm{~cm}$ ).

Of 230 photos recorded, 80 photos had been recorded from a vertical perspective, while the other 150 from tilted perspectives at 60 degree angle. The tilted perspectives had been included, so that the sides of the walls could also be documented.

From image data we generated a point cloud representation and an ortho-photo map of the ruins with a pixel size of $1 \mathrm{~cm}$. Further on, we processed point cloud and generated a digital surface model of the ruins and used image data to generate a textured digital surface model.

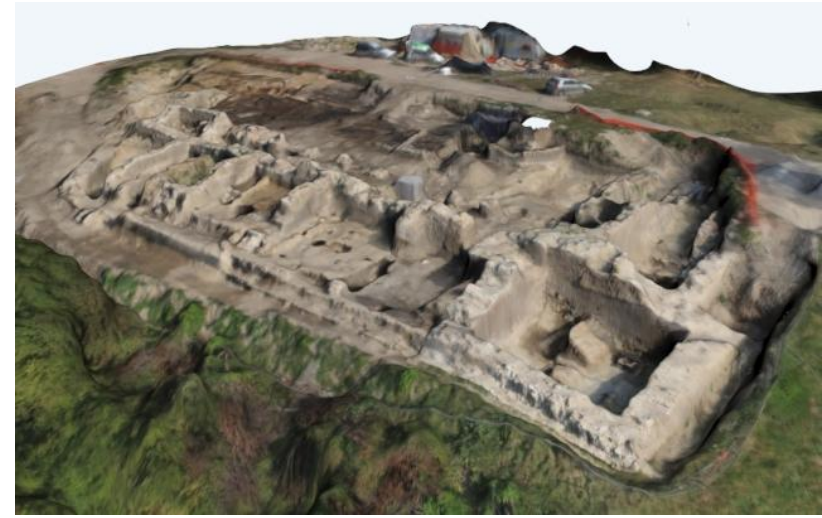

Figure 7. Textured digital surface model of the ruins

\subsection{Preparation of map undelays for planning of infrastructure route}

When detailed map underlay for infrastructure route planning are needed, low altitude photogrammetry can prove to be useful in virtually bring natural environment into the offices of architects and infrastructure-city-land use planners. Orthophoto combined with a point cloud and a DTM offers an illustrative representation of reality.

A new natural gas pipeline is proposed between the towns of Naklo and Podbrezje. To help plan the route of the pipeline, we used our UAV system to generate digital orthophoto map with a pixel size of $3 \mathrm{~cm}$, digital terrain model and a point cloud of the area.

The route of the new pipeline is $10 \mathrm{~km}$ long. We divided the route into 22 parts, which were than individually measured. For each measurement 9 ground control points had been signalized. To cover a greater area in single flight, images had this time been recorded from the altitude of $140 \mathrm{~m}$ with a $66 \%$ overlap. It took us five days of field work to photograph the whole route and another ten days to process images and make ortho-photo maps, point cloud and digital terrain model.

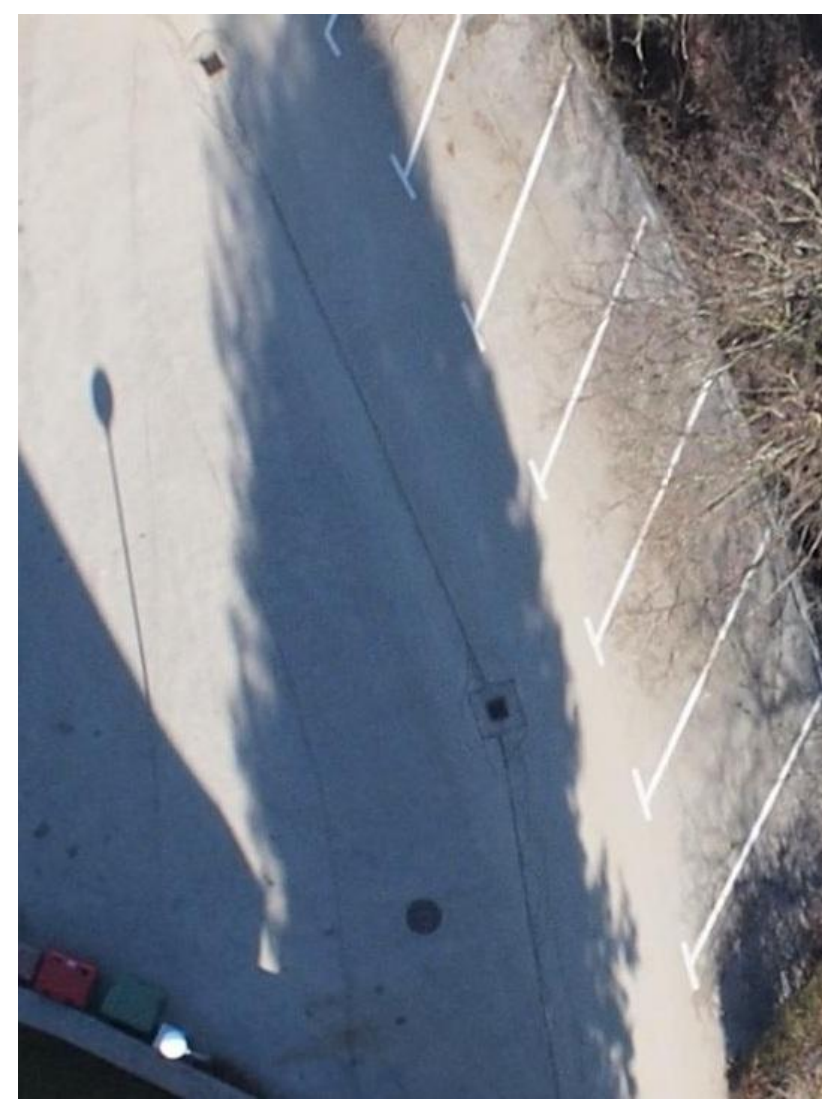

Figure 8. A subset of orthophoto map with ground pixel size of $3 \mathrm{~cm}$

In Figure 8 a subset of orthophoto map is displayed. On the map sewer covers can easily be seen. In this case even the route of underground sewer pipelines can be observed. This kind of information can simplify infrastructure planning process.

\section{ANALYSIS}

We believe low altitude photogrammetry can help fill the gap between classic tachymetric measurements and laser scanning. The technology offers the advantages of both techniques. For the price of classic tachymetric measurements, information can be obtained, which can be competed against that obtained by much more expensive laser scanning.

While experienced surveyors can determine positions of approximately 3 points per minute, can instead laser scanning and low altitude photogrammetry provide millions of points in less than one hour.

Finally these are contactless measurements which allow illustrious representation of natural or manmade environment. We can use them to get information from places which cannot be easily (or safely) accessed, like highways or rocky cliffs. Since the measurements do not interfere with traffic of work processes, can we use low altitude photogrammetry to offer elegant control measurements of quarries, landfills, highways or roads. 


\section{REFERENCES}

Eisenbeiss, H., Lambers, K., Sauerbier, M., 2005.

Photogrammetric recording of the archaeological site of Pinchango Alto (Palpa, Peru) using a mini helicopter (UAV). In: Proc. of the 33rd CAA Conference, Tomar, Portugal.

Furukawa, Y., Ponce, J., 2007. Accurate, Dense and Robust Multi-View Stereopsis. In: Computer Vision and Pattern Recognition, 2007, CVPR 2007, IEEE, pp. 1-8.

Gruen, A., W., 1985, Adaptive least squares correlation: A powerful image matching technique

Niethammer, U., Rothmund, S. and Joswig, M., 2009. UAVbased remote sensing of landslides, In: International Archives of Photogrammetry, Remote Sensing and Spatial Information Sciences, Vol. XXXVIII, Part 5 Commission V Symposium, Newcastle upon Tyne, UK. 2010496

Noah, S., Seitz, S. M., Szeliski, R., 2006. Photo tourism: Exploring image collections in 3D. ACM Transactions on Graphics (Proceedings of SIGGRAPH 2006), 25(3), pp. 835846. 